\title{
Franz Grillparzer: Das Kloster bei Sendomir
}

\section{August Obermayer}

Wenngleich sich die Einsicht, daß Grillparzers Sprachkunst aus dem Bezugsfeld einer spezifisch österreichischen Kulturtradition hervorging, die sich von der Weimarer Tradition und damit der deutschen Klassik deutlich unterschied, nunmehr generell durchgesetzt hat und es daher nicht mehr notwendig erscheint, bei jedweder Beschäftigung mit Grillparzer darauf aufmerksam zu machen, sollten dennoch gewisse Gemeinsamkeiten mit der Weimarer Klassik nicht übersehen werden. So steht Grillparzer durchaus auf dem Standpunkt der deutschen Klassik, wenn er - wie das auch im Briefwechsel zwischen Goethe und Schiller zum Ausdruck kommt ${ }^{1}$ - die Erzählprosa, im besonderen den Roman, für unpoetisch hält. Daher ist es auch nicht weiter verwunderlich, daß Grillparzers literarisches Werk nur sehr wenige Prosatexte umfaßt. Neben Tagebüchern, autobiographischen Schriften und Reiseberichten, die Grillparzer selber wohl kaum als Sprachkunstwerke betrachtet hatte, sind es vor allem die beiden, heutzutage als Novellen bezeichneten Texte Das Kloster bei Sendomir (1827) und Der arme Spielmann (1847). Beide Texte erschienen in einem der im neunzehnten Jahrhundert so beliebten Taschenbücher und Almanache, deren Herausgeber aus verständlichen Gründen Schriftsteller mit bekannten Namen als Beiträger anwarben. Das Kloster bei Sendomir wurde sicherlich von vielen Zeitgenossen als Nebenprodukt des berühmten Dramatikers angesehen. Von der Tageskritik wurde die Novelle nach ihrem Erscheinen zwar nicht unfreundlich aufgenommen, doch jeweils nur mit ein paar Zeilen bedacht. ${ }^{2}$ Betty Paoli berichtet $1875^{3}$, "Das Kloster bei Sendomir schrieb er in Hast und Eile, um Schreyvogel, dem ein ihm von anderer Seite zugesagter Beitrag für die Aglaja ausgeblieben war, aus einer peinlichen Verlegenheit zu helfen". Grillparzer selber bezeichnet dieses Werk in einem Brief an Paul Heyse vom 16. Juni 1870 als "Almanach-Novelle"4, was

1. Vgl. z.B. Schillers Brief an Goethe vom 20. Oktober 1797: "Die Form des 'Meisters', wie überhaupt jede Romanform, ist schlechterdings nicht poetisch, sie liegt ganz nur im Gebiete des Verstandes, steht unter allen seinen Forderungen und partizipiert auch von allen seinen Grenzen." Der Briefwechsel zwischen Goethe und Schiller, hrsg. von Siegfried Seidel, München 1984, Bd. 1, S. 431.

2. Vgl. Franz Grillparzer, Sämtliche Werke, hrsg. von August Sauer, fortgeführt von Reinhold Backmann, Wien 1944, 1. Abt., Bd. 22, S. 41 f.

3. Vgl. Dichter über ihre Dichtungen. Franz Grillparzer, hrsg. von Karl Pörnbacher, München 1970, S. 255.

4. Franz Grillparzer, Sämtliche Werke. Ausgewählte Briefe, Gespräche, Berichte, hrsg. von Peter Frank u. Karl Pörnbacher, München 1965, Bd. 4, S. 875 (Zitiert als $G W$ ). 
nahezu als Gattungsbezeichnung zu verstehen ist. Wie Hellmuth Himmel in diesem Zusammenhang zeigte, erwartete man von einer solchen Novelle, daß sie den Leser fesselte, sein Mitgefühl erregte und in einem ungewöhnlichen Milieu spielte. Angesichts der beschränkten Reisemöglichkeiten wurden jedoch schon Polen, Italien, Schottland und Spanien als exotisch empfunden und befriedigten als Schauplätze den biedermeierlichen Exotismus. ${ }^{5}$

Grillparzers Bestreben war es, "Poesie" hervorzubringen. Er unterschied zwischen "Poesie der Auffassung und Poesie der Darstellung"6 weshalb er dem Roman, für ihn Prototyp der "Poesie der Auffassung", nur den Rang von "halber Poesie" zuzugestehen bereit ist. Den Unterschied von Roman und Novelle sieht Grillparzer darin, daß er den Roman als "Hinaufsteigen der Prosa zur Poesie" betrachtet, während die Novelle für ihn das "Herabsteigen der Poesie zur Prosa"7 bedeutet. Obwohl er auch der Meinung war, daß man jede gute Novelle in Verse bringen könne, da sie "eigentlich ein unausgeführtes poetisches Sujet"8 sei, schreibt er seine beiden Novellen doch in Prosa, äußert aber schon 1843, also vor dem Erscheinen des Armen Spielmann, Adolf Foglar gegenüber:

Es ärgert mich, wenn ein guter Dramatiker in Prosa schreibt [...] Von jeher war der Vers die Sprache der Poesie, und Prosa die der Wirklichkeit. Die Poesie aber will sich eben von der Wirklichkeit entfernen, darum soll sie sich auch im Ausdruck von ihr unterscheiden; nur die Elemente muß sie von ihr nehmen. ${ }^{9}$

Mit dem Terminus "Prosa" bezieht sich Grillparzer dezidiert auf den Roman. Er selbst bekennt, als er von Gustav Heckenast, dem Verleger der Iris, erneut aufgefordert wurde, für den dann nicht zustande gekommenen Jahrgang 1849 einen weiteren Prosabeitrag zu verfassen:

So sehr mich dieses Begehren erfreut, da es beweist, daß Sie mit der heurigen Leistung zufrieden sind, so muß ich nur bemerken, daß Erzählungen überhaupt nicht mein Fach sind $[. . .]^{10}$

5. Hellmuth Himmel, "Grillparzers Novelle Das Kloster bei Sendomir. Struktur und Erzählsituation" in: Grillparzer-Forum Forchtenstein 1976, S. 42.

6. Tgb. 2137 (1934). GW, Bd. 3, S. 291.

7. Tgb. 3476 (1938). GW, Bd. 3, S. 291.

8. Tgb. 3476 (1938). $G W$ Bd. 3, S. 291.

9. Grillparzers Ansichten über Literatur, Bühne und Leben aus Unterredungen mit Adolf Foglar, Wien 1872, S. 29.

10. Reinhold Backmann, "Grillparzer und Stifter in der Iris für 1848" in: Jahrbuch der Grillparzer-Gesellschaft, Neue Folge, Bd. 2 (1942), S. 135. 
Angesichts dieser Sachlage scheint es daher auch nicht verwunderlich, daß Grillparzers Prosa zu Lebzeiten des Autors keinen großen Widerhall fand und auch von der Forschung nicht im selben Maße wie die Dramen beachtet wurde. Nach dem Zweiten Weltkrieg ändert sich diese Situation für den Armen Spielmann zwar grundlegend ${ }^{11}$, für Das Kloster bei Sendomir hat sich jedoch das Klischee vom künstlerisch unbedeutenden und von der Forschung vernachlässigten Werk eingebürgert. Sehr oft geht die neuere Grillparzerliteratur verschämt über das Werk hinweg, als ob es sich um eine Jugendsünde handelte, die zwar der Vollständigkeit halber erwähnt wird, im übrigen aber des großen Dichters keineswegs würdig zu sein scheint. Daß dieser Text aber einem Dichter wie Gerhart Hauptmann nicht zu schlecht war, um ihn zu dramatisieren, wenngleich mit gewissen Veränderungen, hätte zu denken geben sollen und bei näherer Zusicht zeigt sich auch, daß die Sekundärliteratur an Fülle sich zwar nicht mit der zum Armen Spielmann vergleichen läßt, daß aber viel mehr über Das Kloster bei Sendomir gearbeitet wurde, als das tradierte Klischee vermuten läßt.

Bereits die erstmals 1901 und dann in neu bearbeiteter und veränderter Form 1910 erschienene deutsche Fassung von August Erhards in französischer Sprache verfaßten Monographie Franz Grillparzer. Sein Leben und seine Werke ${ }^{12}$ vermittelt ganz grundlegende Einsichten in das Werk. Es wird zwar eingeräumt, daß das Kloster bei Sendomir einen Verteidiger brauchen könne, diese Verteidigung wird dann aber auch geleistet. Der Untersuchung liegt die Analyse einer Verfahrensweise zugrunde, die erst ein halbes Jahrhundert später unter der Bezeichnung "künstlerische Eigenart" Kurswert bekam. Erhard geht davon aus, daß beide Novellen "gewisse Eigentümlichkeiten Grillparzers beleuchten und in klar erkennbarem Zusammenhang mit seinen übrigen Dichtungen stehen." 13 So erkennt er bereits ganz klar, daß in den an Walter Scott gemahnenden Schauplätzen des Klosters bei Sendomir nicht nur der grelle Effekt der Ahnfrau wieder auflebt, sondern auch, daß die Handlung der Novelle bis in Einzelheiten als Vorgeschichte des Trauerspiels fungieren könnte. Weiters verweist Ehrhard auf das Thema der Lüge - Elga täuscht und belügt Starschensky - mit dem sich Grillparzer intensiv in Weh dem, der lügt! und Esther auseinandersetzt, auf das Thema der Ordnung Starschensky ist ein Freund der Ruhe und Ordnung - mit dem sich Grillparzer vor allem im Treuen Diener seines Herrn beschäftigt und stellt eine auffallende

11. Vgl. Grillparzer's "Der arme Spielmann". New Directions in Criticism, hrsg. von Clifford Albrecht Bernd, Columbia 1988. Der Herausgeber stellt in seinem Nachwort sogar die Frage, ob sich wohl die Auffassung, Der arme Spielmann sei Grillparzers perfektestes Werk, der man zum Teil im angelsächsischen Bereich huldigt, auch in Osterreich und damit implizite im deutschsprachigen Bereich, bald durchsetzten werde.

12. August Erhard, Franz Grillparzer. Sein Leben und seine Werke. Deutsche Ausgabe von Moritz Necker, München 1910.

13. August Erhard, a.a.O., S. 446. 
Ähnlichkeit zwischen Starschensky und König Alfons (Die Jüdin von Toledo) fest, asketisch lebender Männer, deren Sinnlichkeit durch eine Berührung mit der Hand entfesselt wird. Von Starschensky heißt es: "Eine warme, weiche Hand ergreift die seinige. [...] Ein bis dahin unbekanntes Gefühl ergriff den Grafen bei der Berührung der warmen Hand. [...] Eine neue Welt stand vor ihm auf, und bebend folgte er seiner Führerin [...]"14, während König Alfons sich ein Liebesabenteuer wie folgt vorstellt:

Da trittst du ein und eine warme Hand

Ergreift die deine, führt dich durch die Gänge,

Die dunkel wie das Grab und endlos gleitend

Den Wunsch erhöhn [...] (V. 466-469) ${ }^{15}$

Ehrhard erkennt bereits die Handschrift des Dramatikers im folgerichtigen Aufbau der Novelle und glaubt den Grund für die Prosabearbeitung des Stoffes darin zu sehen, daß es sich sehr gut um die Vorgeschichte der Ahnfrau handeln könnte und Grillparzer eine Wiederholung der Bühneneffekte der Schauerromantik seit seinem ersten Bühnenerfolg bewußt vermieden hat.

Max Lederer bemüht Das Kloster bei Sendomir als Demonstrationsobjekt für ein von ihm postuliertes "biologisches Prinzip"16 in der Literaturbetrachtung. Er stipuliert lyrische, dramatische und epische Begabungen, die zwar jeweils die Grenzen ihrer Begabung überschreiten können, ihre spezifische Begabung dann aber immer in das ihrer Begabung fremde Genre einbringen. Die Einbringung des dramatischen Elementes in die Novelle, sei nicht unangemessen, weil das Dramatische der Novelle nicht wesensfremd sei. Grillparzers Kloster bei Sendomir sei trotz aller epischen Anlage und Gestaltung doch die Novelle eines Dramatikers, weil "der Charakter Elgas wie hinter einem Schleier verhüllt bleibt"17 und markante Stellen wie dramatische Szenen dargestellt seien.

Leonhard Beriger sieht diese Novelle vor allem im autobiographischen Zusammenhang als jenen Text bei dem sich Grillparzer so sehr hinter seinem Stoff verbirgt wie bei keinem anderen seiner Werke, erkennt in Starschensky, der aus seiner stillen und abgeschiedenen Welt plötzlich in eine neue Welt versetzt wird und an dieser Grenzüberschreitung scheitert, ein "typisches Grillparzerschicksal"18. Für Elga stellt Beriger ebenfalls fest, daß ihr die "Abhängigkeit von

14. $G W$ Bd. 3. Zitiert wird nach dieser Ausgabe.

15. GW Bd. 2, S. 467.

16. Max Lederer, "Die Novelle des Dramatikers" in: Neophilologus 5 (1920), S. 315333.

17. A.a.O., S. 327.

18. Leonhard Beriger, Grillparzers Persönlichkeit in seinem Werk, Horgen-Zürich, Leipzig 1928, S. 81. 
Menschen und Verhältnissen zum Verhängnis"19 wird. Besonders sieht Beriger aber die symbolische Bedeutung der Dunkelheit und der Nacht für diesen Text. Starschenskys Sinne werden durch die Berührung der Hand im dunklen Flur erweckt, wodurch diese Begebenheit schon unter ein ungünstiges Vorzeichen gestellt wird.

1929 widmet Gerhart Reckzeh dem Kloster bei Sendomir ein längeres Kapitel $^{20}$ in dem er ausgehend von Betty Paolis Bericht "überstürzte und unausgereifte Niederschrift" konstatiert, was er vor allem damit begründet, daß sich die Novelle vorwiegend darauf beschränke "Handlung zu geben" und in der "Charakteristik sehr unkonsequent" sei. Reckzeh, der in seiner Untersuchung vor allem nationale Typologien erstellen will, sieht in der Selbsterniedrigung des büßenden Starschensky einen Charakterzug, den er mit dem Charakter eines polnischen Adeligen aus dem 17. Jahrhundert für total unvereinbar hält und kommt zu dem Ergebnis, daß Grillparzer hier wohl eine typisch slawische, nicht so sehr polnische Art charakterisiert habe. Diese Behauptung läßt sich aber nur durch eine etwas angestrengte geistige Akrobatik aufrecht erhalten. Reckzeh ist ehrlich genug zuzugeben, daß Grillparzer die Polen achtete und für Polen dieselbe Mittlerfunktion zwischen Ost und West sah, wie sie auch Österreich erfüllte, modifiziert diese Feststellung aber dahingehend, daß dies Grillparzers bewußte Meinung gewesen sei, im Unterbewußten hätte er sicherlich eine Abneigung gegen die Slawen verspürt und den Polen allgemein slawische Züge verliehen, sodaß sich der deutsch-slawische Gegensatz, wie er sich im Ottokar ausdrücke, im Kloster bei Sendomir fortsetze. Diese nicht sehr ergiebige Fragestellung und dubiose Schlußfolgerung haben in der späteren Forschung keinerlei Nachhall gefunden.

Wolfgang Baumgart setzt sich in seiner Studie ${ }^{21}$ eingehend mit der Quellenfrage auseinander, nimmt aber als gegeben an, daß es sich um eine "sorglos erzählte Novelle" handelt, die als Kunstwerk nicht sehr hoch einzuschätzen sei. Baumgart kann aufgrund seiner Untersuchung und der von ihm aufgespürten Quelle einen Beitrag zur Datierungsfrage leisten. Wie sich zeigt, geht Grillparzers Beschäftigung mit dem Stoff auf das Jahr 1920 zurück. 1922 notiert Grillparzer eine Skizze in seinem Tagebuch, arbeitet die Novelle aber erst 1927 aus, was daraus hervorgeht, daß die von Baumgart eruierte Quelle, das Chronicon slavo-sarmaticum des Procosius erst 1927 im Druck erscheint. Baumgart rechtfertigt die ausführliche Studie eines von ihm selbst als künstlerisch

19. Leonhard Beriger, a.a.O., S. 82.

20. Gerhart Reckzeh, Grillparzer und die Slaven, Weimar 1929, S. 58-70.

21. Wolfgang Baumgart, "Grillparzers Kloster bei Sendomir. Neues zur Quellenfrage, Entstehung und Datierung" in: Zeitschrift für deutsche Philologie 67 (1942), S. 162-176. 
minderwertig bezeichneten Werkes damit, daß "die herausgearbeitete literarische Quelle und ihr Verhältnis zum Werk unser Wissen um die dichterische Technik des Erzählers Grillparzer und die Arbeitsweise des Dichters überhaupt mit der Beobachtung einer geradezu wissenschaftlichen Genauigkeit, die er auch einer schnellen und bestellten Arbeit zuwendet" bereichert. Ob diese Resultate jedoch tatsächlich Einsichten in die dichterische Technik Grillparzers eröffnen, ist fraglich.

Es war dann vor allem Wolfgang Paulsen vorbehalten, sich 1944 in einem Aufsatz mit Grillparzers Erzählungskunst ${ }^{22}$ auseinanderzusetzen und zu einer Zeit, als das noch nicht selbstverständlich war, zu äußern, daß Grillparzer "die deutsche Literatur doch um zwei ihrer hervorragendsten novellistischen Gebilde bereichert" 23 habe. Auch Paulsen beobachtet die stofflichen und thematischen Zusammenhänge des Klosters bei Sendomir mit der dramatischen Produktion Grillparzers, erkennt die dramatische Folgerichtigkeit des Aufbaues und kommt zu dem Ergebnis, daß der Text als "Beichte" des Dichters zu verstehen sei, der sich mit allen ihm zu Gebote stehenden künstlerischen Mitteln bemühte, den Text zu objektivieren und den Charakter der Beichte zu verhüllen. Paulsen liest beide Novellen als Beichten: "Im Spielmann setzte Grillparzer sich mit dem Wert seines Dichtertums auseinander, das er in Frage gestellt glaubte, aber im Kloster machte er sich zum Richter über sein Menschentum, das er in Frage gestellt wußte. ${ }^{24}$ Paulsen selbst untersucht die Kunst des Verhüllens nicht, doch gab er eine wertvolle Anregung, die Früchte hätte tragen sollen. Das geschah aber nicht. In den beiden der deutschen Novelle gewidmeten Bänden von Johannes Klein ${ }^{25}$ und Fritz Lockemann ${ }^{26}$ wird Das Kloster bei Sendomir in einigen wenigen Zeilen abgetan. Johannes Klein merkt zumindest an "die Handlung tritt hervor, die Psychologie verbirgt sich fein." 27

1962 interpretiert Konrad Schaum die Novelle in einer kleinen Studie ${ }^{28}$, in der er sie in den weltanschaulichen Kontext anderer Werke Grillparzers stellt und vor allem einem metaphysisch orientierten Interpretationshorizont verpflichtet ist, der die verallgemeinernde Aussage der Detailanalyse vorzieht. Douglas Yates'

22. Wolfgang Paulsen, "Grillparzers Erzählungskunst" in: Germanic Review 19 (1944), S. 59-68.

23. Ibid., S. 60 .

24. Ibid., S. 67.

25. Johannes Klein, Geschichte der deutschen Novelle von Goethe bis zur Gegenwart, Wiesbaden 1954.

26. Fritz Lockemann, Gestalt und Wandlungen der deutschen Novelle, München 1957.

27. Ibid. S. 157.

28. Konrad Schaum, "Grillparzers Kloster bei Sendomir" in: Wort in der Zeit 8 (1962), Nr. 9, S. 41-45. 
englischsprachige Grillparzerbiographie ${ }^{29}$ behandelt die Novelle zwar auf drei Seiten, kommt aber zu keinen wesentlich neuen Einsichten. Im Jahre 1968 nahm sich Richard $\mathrm{H}$. Lawson des Textes an. ${ }^{30}$ Er postuliert einen kritischen consensus, daß das Werk im Vergleich zum Armen Spielmann als Kunstwerk zweitrangig sei, sieht einen möglichen Grund für diese Beurteilung in der Tatsache, daß Elga als total verworfener Charakter aufgefaßt wurde und bemüht sich um eine Ehrenrettung dieser Figur, indem er sie als eine Vorläuferin des Frauentypus, der seit Schnitzler als "das süße Mädel" bekannt wurde, auszuweisen sucht, um zu dem Schluß zu kommen, daß sie in allem, was sie tut, nur sich selbst treu ist. Mag schon der Versuch einer solchen Typologie etwas verkrampft erscheinen, so überzeugt sein Ergebnis in keiner Weise und entkräftet keineswegs den Vorwurf der Inferiorität in künstlerischer Hinsicht.

Im Grillparzerjahr 1972 setzten im verstärkten Maße Bemühungen um den Autor und sein Werk ein und auch das Kloster bei Sendomir wurde damit bedacht. W. E. Yates, der in seiner kritischen Einführung dem Armen Spielmann ein Kapitel widmet, tut das Kloster bei Sendomir, das er als "minor work"31 bezeichnet, auf einer Seite $a b$, in der er auch keine über die bisherige Forschungslage hinausgehenden Einsichten vorbringt.

Hat sich die Forschung mit wenigen Ausnahmen bisher zurückhaltend bis ablehnend über die künstlerische Qualität des Textes geäußert, bezeichnet Herbert Seidler im Rahmen der Grillparzer-Feier der Österreischischen Akademie der Wissenschaften das Kloster bei Sendomir als den "künstlerische[n] Höhepunkt einer ersten Periode der österreichischen Biedermeiernovellistik" ${ }^{\text {32 }}$ und als "erzählerische[s] Meisterwerk". ${ }^{33}$ Aber selbst Seidler widmet dem Text bloß eine halbe Seite. Da angesichts der Forschungslage erst erwiesen werden müßte, daß es sich um ein "erzählerisches Meisterwerk" handelt, können die vier Punkte, die Seidler als Unterstützung seines Urteils anführt, wohl nur als Anregung für künftige Forscher gelten. Seidler sieht die Bedeutung der Novelle in vierfacher Weise: einmal seien Grillparzers persönliche Wirren, vor allem sein Verhältnis zu Charlotte Paumgarten in die Novelle eingebaut und künstlerisch aufgehoben; dann gestalte Grillparzer einen Menschen der aus der Stille heraustrete und in schuldhafte Verstrickung gerate dadurch, daß er durch Anmaßung eine höhere

\footnotetext{
29 Douglas Yates, Franz Grillparzer: A Critical Biography, London 1964.

30. Richard H. Lawson, "The Starost's Daugther: Elga in Grillparzer's Kloster bei Sendomir" in: Modern Language Association 1 (1968), S. 31-37.

31. W. E. Yates, Grillparzer. A Critical Introduction, Cambridge 1972, S. 164.

32. Herbert Seidler, "Grillparzer als Lyriker, Epigrammatiker und Erzähler" in: Grillparzer-Feier der Akademie 1972. Politik, Gesellschaft, Theater, Weltwirkung. Sitzungsberichte der Österreichischen Akademie der Wissenschaften, phil.-hist. Klasse, Bd. 280, 1. Abh., S. 122.

33. Ibid.
} 
Ordnung störe; die Novelle stehe durch die in ihr vermittelte Atmosphäre des Unheimlichen und Schauerlichen im Zusammenhang mit Grillparzers übrigem Schaffen, besonders mit der Ahnfrau und dem Goldenen Vließ; sie sei ein Kunstwerk von großer architektonischer Meisterschaft und strengem Erzählprinzip. Es handle sich um eine Dichtung von großer Geschlossenheit der künstlerischen Form und einer strengen Begrenzung des Gehalts auf die Gegensätzlichkeit des Guten und Bösen im Menschen. ${ }^{34}$

Winfried Freund betrachtet den Text unter dem Aspekt der Kriminalnovelle und betont neben dem biographischen Hintergrund vor allem den politischen und sozialen Bezug der Novelle zu ihrer Entstehungszeit und kommt zu der Einsicht:

Diese Novelle zeigt mit aller Deutlichkeit, wie die Gesellschaft des Vormärz der Einzelpersönlichkeit in zunehmendem Maße fernrückt, so daß er [sic!] bei wachsendem Mißtrauen keine Chance zur Integration sieht und das Hineinreichen sozialer Konsequenzen in seinen Privatbereich ausnahmslos als tragisch endet. ${ }^{35}$

Richard Allen nimmt die wiederholt in der Forschung vorgebrachten Bemerkungen vom verhüllenden Erzählen ernst und widmet diesem Phänomen eine eigene Untersuchung. ${ }^{36}$ Allen unterscheidet zwischen bewußter Verhüllung durch die Charaktere, das heißt Vorenthalt von Information und bewußte Irreführung, was dann in Verkennung resultiert - z.B. erkennen die beiden deutschen Ritter in dem erzählenden Mönch nicht Starschensky, da dieser sich bemüht seine Identität zu verhüllen - und einer verhüllenden Charakterisierung der Figuren durch den Erzähler des Rahmens, die deutschen Ritter werden als Fremde beschrieben, die am Fenster saßen, im eifrigen Gespräch vertieft "vielleicht vom Zweck ihrer Reise, offenbar von Wichtigem" (S. 120).

In ihrer Studie über Grillparzer als Dichter des sozialen Konflikts widmet Dagmar Lorenz dem Kloster bei Sendomir ein Kapitel ${ }^{37}$ Sie bringt eine neue Sichtweise in die Diskussion und betont vor allem die Rolle der Frau als Opfer, das in einer patriarchalischen Welt immer von anderen definiert wird und nie sich selbst definieren darf. Elga und Oginsky, der den Kampf mit Starschensky verweigert, werden als außerhalb der Welt des männlichen Moral- und Wertgefüges begriffen, denen Leben und Überleben höchste Priorität sind. Lorenz kommt jedoch zu dem Schluß, der Autor stehe auf "seiten des Lebens selbst und kritisch

\section{A.a.O., S. $122 \mathrm{f}$.}

35. Winfried Freund, Die deutsche Kriminalnovelle von Schiller bis Hauptmann, Paderborn 1975, S. 62.

36. Richard Allen, "The Fine Art of Concealment in Grillparzer's Das Kloster bei Sendomir" in: Michigan Germanic Studies 1 (1975), S. 181-188.

37.

Dagmar Lorenz, Grillparzer: Dichter des sozialen Konflikts, Wien, Köln 1986, S. $57-61$. 
gegenüber abstrakten und weltfremden Konventionen, die es zerstören" ${ }^{38} \mathrm{Daß}$ der falsche Mönch Starschensky nicht darauf angelegt sei, beim Leser Sympathien zu erwecken, dies bei seinen fiktiven Zuhörem jedoch tut "muß Skepsis erregen an der Gesellschaft, in der das mörderische Prinzip auf Sympathien stößt." ${ }^{39}$ Hier berührt sich Dagmar Lorenz mit einer der Grundthesen Zdenko Skrebs, der sich in seiner Studie ${ }^{40}$ bemüht, Grillparzer als einen "Dichter der Jugend und des Eros" darzustellen.

Wenngleich es letzthin für die Interpretation eines Textes unerheblich ist, welche biographischen Fakten als Anlaß, Stoff, Thema, Motiv oder Inspiration in Frage kommen, kann solches Material immerhin wertvolle Hinweise für eine Interpretation liefern, die freilich dann immer am Text bestätigt werden müssen. Pläne für das Kloster bei Sendomir gehen bis zum Jahre 1820 zurück, einer Zeit also, in der Grillparzer ein Verhältnis mit Charlotte Paumgarten, der Frau seines Cousins unterhielt. Grillparzer hätte sich demnach in Oginsky dargestellt. 1823 beginnt Grillparzer aber ein Verhältnis mit Marie von Smolenitz, an der auch der Maler Daffinger Interesse zeigte. Grillparzer fühlte sich hier von seinem Freund Daffinger betrogen, der sich auch als der Vater von Maries Kind entpuppte, das 1926 geboren wurde, was Grillparzer die Rolle Starschenskys zuweisen würde. Der Tod Charlottes, der erst nach Publikation der Novelle eintrat, erscheint hier vorweggenommen. Natürlich ist die Biographie in der Regel nicht in jeder Einzelheit in Dichtung übertragen, doch wurde schon früh bemerkt, daß es sich bei diesem Text um eine Beichte Grillparzers handelt, die aber aus offensichtlichen Gründen nicht zu deutlich sein durfte und daher in verhüllter Form erscheint. Diese Verhüllung wurde in der Forschung wiederholt moniert, Richard Allan widmet diesem Kunstprinzip einen Aufsatz. Es will aber scheinen, daß dieses Prinzip nicht ganz ausgeschöpft wurde. Es handelt sich bei diesem Text, wie in der Folge gezeigt werden soll, um schrittweise Enthüllung durch Verhüllung. In der konsequenten Durchführung dieses Prinzips scheint die künstlerische Bedeutung dieses Textes zu liegen. Hier wird ein Erzählprinzip angewendet, welches, zumindest an der Oberfläche betrachtet, aller literarischen Konvention zuwiderläuft. Ist der Zweck literarischen Schaffens Mitteilung und Kommunikation durch bestimmte sprachliche Modalitäten, scheint sich hier geradezu das Nichtmitteilen als Prinzip etabliert zu haben. Wie aber dennoch Kommunikation erreicht werden kann, ist das hier zu bewältigende künstlerische Problem.

Bereits im Rahmen deutet sich dieses Prinzip an. Der Rahmenerzähler verhält

38. A.a.O., S. 61.

39. A.a.O., S. 61 .

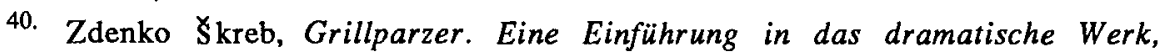
Kronberg/Ts. 1976. 
sich ähnlich wie der Erzähler der Binnengeschichte. Zwei "Fremde" reisen, wie es heißt, durch die Täler der Woiwodschaft Sendomir. Diese Apostrophierung der reisenden Ritter als Fremde, kann aber doch nur meinen, daß es sich nicht um Ortsansässige handelt und daß sie dem Leser fremd sind, erweckt aber den Eindruck, als ob sie auch dem Erzähler fremd wären, obwohl dieser sich in der Lage sieht mitzuteilen, daß es sich um Deutsche handelt, die als Boten des deutschen Kaisers an den Hof des Königs Sobiesky ziehen, Fremde, die von der weiten Welt der großen Politik, von den sonnenvergoldeten Tälern in die Enge und Dunkelheit des Klosters geraten, in der sich ihnen ein privates Schicksal offenbart, nur um am Ende der Geschichte diese Enge des Raumes und des Privaten wieder zu verlassen und in die Weite der Welt und des politischen Geschehens zu ziehen. Diese Rahmenhandlung zeigt aber nicht nur eine temporäre Einengung der Perspektive, die sich nach Ende der Novelle wieder ausweitet und somit eine für das 17. Jahrhundert wie auch für das 19. Jahrhundert typische "Spannung von innerer Befriedung und den Ansprüchen einer unruhigen Außenwelt" 1 signalisiert, es zeigt sich auch ein Erzähler am Werk, der absichtlich verheimlicht und verhüllt. Ein Erzähler, der nicht nur weiß, daß es sich um Boten des deutschen Kaisers handelt, die nach Warschau ziehen, sondern, der auch weiß, daß die beiden sich vorgenommen hatten, auf ihrem Rückweg mehr über den sonderbaren Mönch zu erfahren, jedoch ihre Pläne ändern mußten und nie "mehr etwas von dem Mönche und dem Kloster bei Sendomir gehört" (S. 145) haben, gibt nun vor, in einer Situation, in welcher sich diese Fremden dem Leser enthüllen könnten, nicht zu wissen, wovon sie sprachen "[...] noch immer saßen die beiden Ritter am Fenster, im eifrigen Gespräch; vielleicht vom Zweck ihrer Reise, offenbar von Wichtigem". (S. 120) Das kann doch nur heißen, daß der Erzähler nicht bereit ist, alles mitzuteilen, was er weiß. Ähnlich verfährt der Erzähler mit der sonderbaren Mönchsgestalt. Es scheint als ob der Erzähler besondere Anstrengungen unternähme durch genaue Beschreibung des Äußeren und des Benehmens dieses Mönches möglichst viel über den Charakter dieser Figur mitzuteilen, doch zeigen alle diese detailierten Beschreibungen die Perspektive der staunenden und beobachtenden fremden Ritter. Der Mönch wird eingeführt mit den Worten "[...] eine seltsame Menschengestalt trat ein, mit der Frage: ob sie Feuer bedürfen?" (S. 120) Es wäre nun ohne weiteres möglich gewesen den ganzen folgenden Paragraphen auszulassen und mit der Zeile: "Die beiden sahen sich an, erstaunt ob der seltsamen Erscheinung." (S. 121) fortzufahren. Anstatt dessen fügt der Erzähler aber eine siebzehnzeilige Beschreibung dieser Figur ein:

Der Eingetretene war in ein abgetragenes, an mehreren Stellen geflicktes Mönchskleid gehüllt, das sonderbar genug gegen dem derben, gedrungenen

41. Winfried Freund, Die deutsche Kriminalnovelle von Schiller bis Hauptmann, Paderborn 1975, S. 56. 
Körperbau abstach. Obgleich von Alter scon etwas gebeugt und mehr unter als über der Mittelgröße, war doch ein eigener Ausdruck von Entschlossenheit und Kraft über sein ganzes Wesen verbreitet, so daß, die Kleidung abgerechnet, der Beschauer den Mann eher für alles, als für einen friedlichen Sohn der Kirche erkannt hätte. Haar und Bart, vormals augenscheinlich rabenschwarz, nun aber überwiegend mit Grau gemischt und, trotz ihrer Länge, stark gekräuselt, drängten sich in dichter Fülle um Stirne, Mund und Kinn. Das Auge, klösterlich gesenkt, hob sich nur selten; wenn es aber aufging, traf es wie ein Wetterschlag, so grauenhaft funkelten die schwarzen Sterne aus den aschfahlen Wangen, und man fühlte sich erleichtert, wenn die breiten Lider sie wieder bedeckten. So beschaffen und so angetan, trat der Mönch, ein Bündel Holz unter dem Arme, vor die Fremden hin, mit der Frage: ob sie Feuer bedürften. (S. 120).

Am Ende dieses Paragraphen wird die Frage wiederholt und so der Anschluß an den durch die Detailbeschreibung unterbrochenen Handlungsverlauf wiederherstellt: "[...] so angetan, trat der Mönch, ein Bündel Holz unter dem Arme, vor die Fremden hin, mit der Frage: ob sie Feuer bedürfen?" (S. 120) Wenngleich hier der Erzähler doch einiges über den Mönch mitteilt, erfährt der Leser, wenn auch ziemlich detailiert, nur das, was die beiden Fremden selber beobachten konnten, in jedem Fall aber genug, um im Leser dieselbe Neugierde zu wecken, mehr über das Kloster und den Mönch zu erfahren, die sich bei den beiden Fremden eingestellt hat. Der anonyme Leser wird mit den Fremden identifiziert. Vor Fremden kann man sich offenbaren, allerdings mit der nötigen Vorsicht. Der Mönch als Erzähler der Binnennovelle beginnt seine Erzählung mit den Worten: "Starschensky hieß der Mann [...]" (S. 123) erzählt konsequent in der dritten Person und wird erst nach Beendigung seiner Geschichte durch den mit den Worten "Wo bleibst du, Starschensky?" (S. 145) in das Zimmer der Fremden tretenden Abt entlarvt. Das heißt aber, der Erzähler der Binnennovelle verhält sich ähnlich wie der Erzähler des Rahmens. Er ist nicht gewillt, alles mitzuteilen, was er weiß. Vorsicht und selektives Mitteilen charakterisieren die nun folgende Erzählung, doch gelingt es dem Erzähler nicht, dieses Prinzip konsequent durchzuhalten. Die nicht-verbalen Äußerungen des Binnenerzählers scheinen sich einer derartigen Kontrolle zu entziehen. So reagiert der Mönch auf die Frage der Fremden, welch gottgeliebter Mann denn das Kloster gestiftet hätte, mit einem wahrhaft furiosen Ausbruch:

Da brach der Mönch in ein schmetterndes Hohngelächter aus. Die Stuhllehne, auf die er sich gestützt hatte, brach krachend unter seinem Druck zusammen; eine Hölle schien in dem Blicke zu flammen, den er auf die Fremden richtete, und plötzlich gewendet, ging er schallenden Trittes zur Türe hinaus. (S. 121f.)

In der Binnenerzählung schwankt der Erzähler ständig zwischen der selbst 
auferlegten Distanz und Objektivität, als Technik des Verhüllens und dem Durchbrechen dieses Prinzips, was sich in der Vermittlung intimster Bereiche der Psyche der erzählten Person äußert. Einerseits erzählt er nur Vermutungen und Gerüchte über Starschensky, dann nämlich, wenn selbst durch die Maskierung der dritten Person gewisse Aussagen dem Erzähler die Peinlichkeit der Situation dessen, der sein Eigenlobes singt, deutlich vor Augen führen würden:

Im Kriege hieß man ihn tapfer; sonst lebte er still und abgeschieden im Schlosse seiner Väter. Über eines wunderten sich die Leute am meisten: nie hatte man ihn einem weiblichen Wesen mit Neigung zugetan gesehen, sichtlich vermied er den Umgang mit Frauen. Er galt daher für einen Weiberfeind (S. 123)

Wenn es aber gilt, diese Verhaltensweisen zu erklären oder zu rechtfertigen, weiß der Erzähler plötzlich um die intimsten Gedanken und Gefühle seiner Figur genau Bescheid:

ein über alles gehendes Behagen am Besitz seiner selbst, hatte ihm bis dahin keine Annäherung erlaubt. Abwesenheit von Unlust war ihm Lust. (S. 123)

Während des Erzählens von der ersten Begegnung Starschenskys mit Elga gerät der Erzähler geradezu in Ekstase. Die Begegnung mit dem Mädchen im Dunkel der Stadt, der Ausbruch der Sinnlichkeit durch die Berührung der Hände im dunklen Flur, die Beschreibung von Elgas Schönheit, die "schwarzen Locken", die "gleichgefärbten Wimpern", die "hellblau strahlenden Augen", der Mund mit den "üppig aufgeworfenen beinahe zu hochroten Lippen", Kinn, Wangen, Stirn, Nase alles in vollendetem Einklang mit der "Form eines zugleich schlank und voll gebauten Körpers" wird mit solchem Elan und solcher Selbstvergessenheit vorgetragen, daß der Erzähler für einen kurzen Moment vergißt, daß er die Maske der dritten Person trägt und in die erste Person überwechselt: "Stirn und Nase, wie vielleicht gerade der Maler sie nicht denkt, wie sie aber meinen Landsmänninnen wohl stehen." (S. 124)

Der Mönch wird sich aber dieser seiner Erzählprätention völlig ungemäßen Schwärmerei bewußt, Vorsicht und Maske gewinnen wieder die Oberhand und der Erzähler bremst sich gleichsam selbst mit der Bemerkung: "Ja, ja, bei dem alten Mönch rappelts einmal wieder." (S. 124)

Der Mönch setzt seine Erzählung nun wieder mit größerer Distanz, jedoch deutlich aus der Perspektive Starschenskys fort. Als er aber von der dunklen Gestalt erzählt, auf đie Starschensky durch seinen Hausverwalter aufmerksam gemacht wird, und erfährt, daß diese Gestalt "nachts" das Schloß umschleicht, weiß der Erzähler auf einmal wieder ganz genau über Starschenskys innere Regungen Bescheid: "Starschensky schauderte unwillkürlich zusammen bei dieser 
letzten Antwort [...]" (S. 132) scheint aber nach geraumer Zeit wieder zu merken, $\mathrm{da} B$ er Gefahr laufen könnte seine Maske zu lüften und distanziert sich wieder, indem er vorgibt, nicht zu wissen, wie Starschensky nach dem Auftritt mit Elga die Nacht verbrachte: "Wie er die Nacht zubrachte; wer kann es wissen? Der Diener, der des Morgens zu ihm eintrat, fand ihn angekleidet, auf einem Stuhle sitzend." (S. 134). Unmittelbar darauf nimmt er wieder die Attitüde des allwissenden und kommentierenden Erzählers ein:

Der Graf bestrebte sich nicht bloß, über die Vorfälle des gestrigen Tages nichts zu denken, er dachte wirklich nichts. Denn wenn der verfolgte Strauß sein Haupt verbirgt und wähnt, sein Nichtsehen der Gefahr sei zugleich ein Nichtdasein derselben, so tut der Mensch nicht anders. Unwillkürlich schließt er sein Auge vor einem hereinbrechenden Unvermeidlichen, und jedes Herz hat seine Geheimnisse, die es absichtlich verbirgt vor sich selbst. (S. 135)

Das hindert ihn aber nicht daran, bei Bedarf aus der Perspektive des Hausverwalters zu erzählen: "Das Kind stand zwischen seinen Knieen, er selbst hielt ein Bild in der Hand, abwechselnd auf dieses, dann auf die Kleine blickend, wie einer, der vergleicht, meinte der alte Mann." (S. 136) oder die Existenz von Gerüchten vorzutäuschen, wenn er in Gefahr gerät seine Identität zu verraten:

All diese Geheimnisse soll einer von Elgas Brüdern, gegen den er sich zur rechten Zeit freigebig zeigte, dem Grafen für Geld verraten und ihm zugleich den Ort angezeigt haben, wo Oginsky, einem geleisteten Schwur zufolge, sich verborgen hielt. (S. 137)

So zeigt sich, daß der Erzähler immer wieder in Gefahr gerät, sein Geheimnis zu verraten und daher unter dem Druck, die Fiktion aufrechtzuerhalten, immer wieder nach neuen Möglichkeiten der Distanzierung suchen muß, die zwar manchmal der Logik widersprechen, für die Zuhörer oder Leser jedoch ihre Dienste leisten. Auf der einen Seite sieht sich der Erzähler genötigt sein Wissen um die Umstände der Gefangennahme Oginskys zu verleugnen:

Unvermutet erschien der Graf nach einiger Zeit auf der Grenze seiner Besitzung, in seinem Gefolge ein verschlossener Wagen, von dessen Inhalt niemand wußte. Eine verhüllte Gestalt, vielleicht durch Knebel am Sprechen verhindert, ward herausgehoben [...] (S. 137)

um sich dann aber über eine private Aussprache zwischen Starschensky und Elga, die ohne Zeugen stattgefunden hat, genauestens informiert zu zeigen: "Nun, 
Oginsky! antwortete sie, und ein leichtes Zucken in ihren Zügen verriet eine vorïbergehende Bewegung." (S. 138)

Sogar die Sicht aus Elgas Perspektive wird vom Erzähler nicht verschmäht: "Elga schlief in ihrem Zimmer. Da fühlte sie sich angefaßt und, aus dem Schlafe emporfahrend, sah sie beim Schein der Nachtlampe ihren Gatten [...]" (S. 140). Dieses dauernde Durchbrechen des Prinzips der Verhüllung führt aber schließlich dazu, daß am Ende der Erzählung die Fiktion nicht mehr aufrecht zu halten ist und dem Erzähler die Selbstenthüllung nur durch den eintretenden Abt erspart wird.

Ausgehend von Hinweisen, daß biographische und psychologische Faktoren oder vielleicht gar die Realität der Zensur für diese Art des verhüllenden Erzählens verantwortlich gemacht werden können, deren sich sowohl der Rahmenerzähler als auch der Erzähler der Binnennovelle bedient, konnte am Text gezeigt werden, daß ein solches Bemühen des Erzählers, die Dinge zu verschleiern und zu verhüllen evident ist, was aber nicht konsequent durchgehalten werden kann, sonst würde ja am Ende keine Erzählung zustandekommen. Es läßt sich daher für die Binnenerzählung feststellen, daß die angestrebte objektivierende, auktoriale Erzählhaltung ständig durchbrochen wird, daß es keine einheitliche Erzählperspektive gibt, sondern diese in mehrere personale Perspektiven aufgesplittert ist und daß schließlich der Rahmenerzähler eingreift, der diese für das 19. Jahrhundert doch ziemlich chaotisch anmutende Erzählung zu einem Ende bringt und zusammenhält. Daß diese Aufspaltung der Erzählperspektive für manche Zeitgenossen etwas schockierend gewesen sein mag, ist verständlich. Dieses Unverständnis mag sogar zum Teil für die Berichte, die Erzählung sei in großer Hast und Eile auf Bestellung niedergeschrieben worden, verantwortlich sein. Dieser These hat aber schon Reinhold Backmann widersprochen, der berichtet, daß sich in der Handschrift keine Anzeichen von Hast und Eile feststellen lassen und auch bezweifelt, daß Grillparzer unter Druck auf Bestellung hätte arbeiten können. ${ }^{42}$ Mögen Grillparzers Zeitgenossen dieser Art des Erzählens etwas hilflos gegenübergestanden sein, dem Leser des 20. Jahrhunderts bietet eine derartige Erzähltechnik nicht die geringsten Schwierigkeiten. Im Gegenteil, die Verabschiedung des auktorialen Erzählers und die Einführung der Multiperspektive ist geradezu als ein Konstituent der Moderne bezeichnet worden. Dem Dramatiker Grillparzer hat man wiederholt bescheinigt, ein Vorläufer der Moderne zu sein. Es wäre hiermit zu überlegen, ob Grillparzer nicht für die Erzählprosa ähnliches geleistet hat und man diese Erzählweise nicht durch Eile und Hast entschuldigen sollte, wie es den Zeitgenossen nötig erschien, sondern darin künstlerische Absicht und eine Vorwegnahme künftiger Entwicklungen erkennen sollte.

42

HKA, Abt. 1, Bd. 13, S. 295. 